74.20.Mn, 74.20.-z

\title{
The breakdown of the mean-field description of the Nagaoka phase
}

\author{
Fabio L. Braghin and Alvaro Ferraz \\ International Center for Condensed Matter Physics, Universidade de Brasilia, \\ Caixa Postal 04513, 70904-970 Brasilia, DF, Brazil
}

\author{
Evgueny A. Kochetov \\ Laboratory of Theoretical Physics, Joint Institute for Nuclear Research, 141980 Dubna, Russia \\ International Center for Condensed Matter Physics, Universidade de Brasilia, \\ Caixa Postal 04513, 70904-970 Brasilia, DF, Brazil
}

\begin{abstract}
We discuss the relevance of the improved mean-field slave-fermion theory to describe the Nagaoka $(U=\infty)$ limit of the Hubbard model. In this theory the crucial on-site constraint of no double electron occupancy is taken into account rigorously prior to the mean-field approximation. At oneloop approximation the effective mean-field action shows a long-range ferromagnetic order over the whole doping range. This indicates that the slave-fermion mean-field theory does not constitute an appropriate framework to describe the physics of the Nagaoka phase. We discuss the drawbacks of this mean-field theory and present some results on the derivation of a low-energy effective spin action to describe the Nagaoka phase beyond the mean-field approximation.
\end{abstract}

\section{INTRODUCTION}

It is now widely believed that the essential physics of strongly correlated lattice electrons is encoded in the local constraint of no double electron occupancy. This constraint prohibits double electron occupancy of a lattice site due to the large local Coulomb repulsion between hopping electrons. The on-site Hilbert space is thus restricted to states with at most one electron per site. Such a modification of the underlying Hilbert space results in dramatic consequences for the low-energy properties of the relevant electron systems revealing a rich and unusual physics in this limit.

Formally, given a local electron operator $c_{i \sigma}$ with the spin projection $\sigma=\uparrow, \downarrow$, the local no double occupancy (NDO) constraint reads

$$
\sum_{\sigma} c_{i \sigma}^{\dagger} c_{i \sigma} \leq 1
$$

Let us denote by $\tilde{c}_{i \sigma}$ the projected electron operators that satisfy this requirement. Since the inequality (1) is hard to deal with analytically, one can try to circumvent this difficulty by turning Eq.(11) into an equality at the expense of the introduction of some redundant degrees of freedom. The two well-known ways to accomplish this are the so called slave-fermion (SF) and slave-boson (SB) representations of the constraint electron operator. Namely, by introducing the "slave boson" poses the on-site constraint electron operator in the form,

$$
\tilde{c}_{i \sigma}=b_{i}^{\dagger} f_{i \sigma},
$$

where $b_{i}$ is a charged spinless boson (holon), while $f_{i \sigma}$ ia a neutral, spin $1 / 2$ fermion operator (spinon) satisfying the NDO constraint

$$
b_{i}^{\dagger} b_{i}+\sum_{\sigma} f_{i \sigma}^{\dagger} f_{i \sigma}=1 .
$$

Alternatively, one can also introduce a spinless fermion $f_{i}$ to describe the charge degree of freedom and a spinful boson $b_{i \sigma}$ to keep track of the spin degree of freedom. This is the "slave fermion" approach ${ }^{2}$,

$$
\tilde{c}_{i \sigma}=b_{i \sigma} f_{i}^{\dagger} \text {. }
$$

The NDO constraint now reads

$$
f_{i}^{\dagger} f_{i}+\sum_{\sigma} b_{i \sigma}^{\dagger} b_{i \sigma}=1
$$

The electron spin operator, $\overrightarrow{Q_{i}}=\frac{1}{2} \sum_{\sigma \sigma^{\prime}} \tilde{c}_{i \sigma}^{\dagger} \vec{\tau}_{\sigma \sigma^{\prime}} \tilde{c}_{i \sigma^{\prime}}$, takes in this representation the form

$$
\overrightarrow{Q_{i}}=\frac{1}{2} \sum_{\sigma \sigma^{\prime}} b_{i \sigma}^{\dagger} \vec{\tau}_{\sigma \sigma^{\prime}} b_{i \sigma^{\prime}}
$$

where Eq.(5) has been used. Here $\vec{\tau}^{\prime}$ s are the Pauli matrices.

There is an apparent $\mathrm{U}(1)$ gauge redundancy in the decompositions of the projected electron operator as given by Eqs.(24). Namely, the local gauge transformation generated by the NDO constraint

$$
f_{i} \rightarrow f_{i} e^{i \theta_{i}}, b_{i} \rightarrow b_{i} e^{i \theta_{i}}
$$

keeps the representations (2) and (4) intact. The slaveparticle gauge theory is infinitely strong coupled since there is no kinetic energy for the bare gauge field. Consequently, one should exercise some care in applying an ad hoc mean-field (MF) approximation to treat the slaveparticle theories. Such an approach can only be justified provided that the low-energy gauge coupling gets effectively renormalized to some finite and, potentially, weak coupling. However, this seems hardly to be the case in the slave-particle theories ${ }^{3}$, which in turn casts some doubts on the results obtained within the MF slave-particle theories. Indeed, both the SF and SB theories should in principle produce physically identical results for the t-J model 
of strongly correlated electrons, however they give in the MF approximation very different phase diagrams 4.5 .

The strong electron correlations are at work to full extent in the so called Nagaoka $(U=\infty)$ limit of the Hubbard model. Indeed, in this case an infinitely strong Coulomb repulsion strictly prohibits the double electron occupancy of the lattice sites, and the NDO constraint becomes of the utmost importance. In the infinite $U$ limit, the Hubbard Hamiltonian reduces to

$$
H=-\sum_{i j, \sigma} t_{i j} \tilde{c}_{i \sigma}^{\dagger} \tilde{c}_{j \sigma}+\mu \sum_{i \sigma}\left(1-\tilde{c}_{i \sigma}^{\dagger} \tilde{c}_{i \sigma}\right),
$$

where $t_{i j}$ is a symmetric matrix whose elements represent the hopping amplitude $t$ between the nearest-neighbor sites and are zero otherwise. We have also introduced the chemical potential $\mu$ to control the total number of vacancies (holes). Despite its seemingly simple form, this Hamiltonian cannot be diagonalized due to the fact that the projected electron operators fulfill complicated commutation relations resulting from the explicit manifestation of strong correlations.

Since the Coulomb repulsion $U$, whatever large it may be, is in practice always finite, the one band $U=\infty$ Hubbard model is basically a toy model and does not describe any specific material ${ }^{6}$. Nevertheless, it captures an extreme limit for the physics of strong electron interactions. Had the projected electron operators in (8) been replaced by their related conventional operators, the model would have been reduced to a system of noninteracting electrons revealing thereby a trivial physics. On the other hand, the physics behind the model (8) is certainly far from trivial. Indeed, Nagaoka ${ }^{8}$ proved a theorem stating that for one hole the ground state of the $U=\infty$ Hubbard model is a fully saturated ferromagnet. This provides an interesting example of a quantum system in which ferromagnetism appears as a purely kinetic energy effect with hole hoping (itinerant ferromagnetism) emerging as a result of the strong correlations from the NDO constraint.

Unfortunately, despite extensive work over many years, this model and itinerant ferromagnetism are still poorly understood. One of the important questions to be addressed concerns the thermodynamic stability of the Nagaoka phase. That is, whether or not the Nagaoka state is stable when the density of holes is finite in the thermodynamic limit. There are arguments both for $9,10,11,12,13,14$ and against $15,16,17,18,19$ the thermodynamic stability of the Nagaoka phase and comparisons between various approaches have been made carefully (for a recent example, $\left.\mathrm{see}^{20}\right)$. The basic problem that prevents one from reaching a definite conclusion on that is the large- $U$ limit or, equivalently, the local NDO constraint which is very difficult to deal with in a controlled way. For example, the numerical studies of this problem, although well developed for the Hubbard model, cannot incorporate the large- $U$ limit ${ }^{25}$. Analytical approaches, on the other hand, basically imply a MF treatment in which the local NDO constraint is uncontrollably replaced by a global condition.
In this sense, it is interesting to note, that there are several works considering different variational approaches $9.11,19,21,22,23,24$ in which the NDO constraint is automatically built into the trial state vector. In general, the following observation holds: variational estimations that involve more realistic refined trial wavefunctions result in a smaller value of the critical hole concentration.

One may therefore think that a straightforward application of the MF theory that treats electron correlations on average is not likely to produce reliable results. Specifically, in the present paper we show that the treatment of the Nagaoka phase within the MF SF approximation results in the qualitatively incorrect conclusions.

We outline below the main motivation of this work. The standard SF MF theory which treats the NDO constraint only on average is known to predict a stable FM phase for the $U=\infty$ Hubbard model over a finite though unphysically large doping range. Since the local electron correlations encoded in the NDO constraint are surely of the utmost importance, if one attempts to deal with the properties of this model in a correct and reliable way, one would naturally think that a proper treatment of the constraint prior to the MF approximation could improve that MF result shifting the critical hole concentration towards a much smaller and physically reasonable value. In this paper we show that this is not the case. On the contrary, the improved MF theory predicts a stable FM phase over the whole doping range.

Two main routes which redirects of this result are discussed in Sections II and III. We argue that the finite critical hole concentration predicted by the standard MF theory is just an artifact of the uncontrolled treatment of the NDO constraint. Moreover, the theory based on the MF treatment of the spin degrees of freedom affects the physics of the problem in a qualitative way. Not only the NDO constraint needs to be fulfilled. It is clearly very important to treat the electron correlations encoded by that constraint in a proper way considering both charge and spin degrees of freedom on equal footing as dynamical rather than the MF variables. Some preliminary results in this direction are reported in Section IV.

\section{MF SF TREATMENT OF THE NDO CONSTRAINT}

In this section we briefly go over the conventional MF SF theory of the ferromagnetic phase in the $U=\infty$ Hubbard model as given by, e.g., Boies et a 26 .

To set up the stage, let us start with the SF representation of the projected electron operators given by Eqs.(45). The Hamiltonian (8) can be then rewritten in the form

$$
H=\sum_{i j, \sigma} t_{i j} f_{j}^{\dagger} b_{i \sigma}^{\dagger} b_{j \sigma} f_{i}+\mu \sum_{i} f_{i}^{\dagger} f_{i},
$$

where Eq. (5) has been used. The partition function 
takes the form

$$
Z=\int D \lambda D \bar{\psi} D \psi \prod_{\sigma} D \bar{z}_{\sigma} D z_{\sigma} e^{S(\psi, z, \lambda)},
$$

with the action $S=\int_{0}^{\beta} d \tau \mathcal{L}_{S F}(\tau)$ and the Lagrangian

$$
\begin{aligned}
\mathcal{L}_{S F}(\tau) & =\sum_{i j} \bar{\psi}_{j}\left[\left(-\partial_{\tau}-\mu+\lambda_{i}\right) \delta_{i j}-t_{i j} \sum_{\sigma} \bar{z}_{i \sigma} z_{j \sigma}\right] \psi_{i} \\
& +\sum_{i \sigma} \bar{z}_{i \sigma}\left(-\partial_{\tau}+\lambda_{i}\right) z_{i \sigma}-\sum_{i} \lambda_{i} .
\end{aligned}
$$

Here $z_{i \sigma}$ and $\psi_{i}$ stand for complex numbers and complex Grassmann parameters, respectively. The purely imaginary field $\lambda_{i}$ has been introduced to enforce the local NDO constraint,

$$
\bar{\psi}_{i} \psi_{i}+\sum_{\sigma} \bar{z}_{i \sigma} z_{i \sigma}=1
$$

In this representation the electron spin operator (6) becomes

$$
\vec{Q}_{i}=\frac{1}{2} \sum_{\sigma \sigma^{\prime}} \bar{z}_{i \sigma} \vec{\tau}_{\sigma \sigma^{\prime}} z_{i \sigma^{\prime}}
$$

\section{A. Zeroth-order approximation}

At the zeroth-order MF approximation boson variables take a macroscopic value and all fluctuations in space and imaginary time are neglected, i.e.

$$
z_{i \sigma}(\tau)=z_{\sigma}^{(0)}, \quad \lambda_{i}(\tau)=\lambda^{(0)} .
$$

Under this condition, the fermionic degrees of freedom in (10) can easily be integrated out to yield for the free energy $\mathcal{F}:=-\frac{1}{N \beta} \log Z$,

$$
\begin{aligned}
\mathcal{F}_{S F} & =-\frac{1}{N \beta} \sum_{\vec{k}} \log \left(1+e^{-\beta\left(E(\vec{k})+\mu-\lambda^{(0)}\right)}\right) \\
& -\sum_{\sigma}\left|z_{\sigma}^{(0)}\right|^{2} \lambda^{(0)}+\lambda^{(0)}-\mu \delta
\end{aligned}
$$

where

$$
E(\vec{k})=\left(\sum_{\sigma}\left|z_{\sigma}^{(0)}\right|^{2}\right) t_{\vec{k}}
$$

and $\delta$ is the average density of holes. The ground-state saddle point equations determining $z_{\sigma}^{(0)}, \mu$ and $\lambda^{(0)}$ describe a saturated ferromagnet, $m \propto(1-\delta)$, where the average magnetization is given by $m=\frac{1}{2} \sum_{\sigma} \sigma\left|z_{\sigma}^{(0)}\right|^{2}$. Such a solution exists over the whole doping range, $0 \leq \delta<1$.

It is interesting to note that due to the global $\mathrm{MF}$ constraint

$$
\delta+\sum_{\sigma} \bar{z}_{\sigma}^{(0)} z_{\sigma}^{(0)}=1
$$

the spin variables $z_{\uparrow}^{(0)}$ and $z_{\downarrow}^{(0)}$ cannot vanish simultaneously. This observation rules out the possibility of having, within that approach, a phase transition into a disordered phase by identifying the set of macroscopic spin amplitudes, $z_{\sigma}^{(0)}$, as the relevant order parameter.

\section{B. One-loop approximation}

The one-loop correction to the effective action can in principle alter this result destabilizing the zeroth-order MF theory at some critical hole concentration, $\delta_{c}$. In the MF SF theory the one-loop approximation takes the form 26 :

$$
z_{q}^{\sigma}=z_{\sigma}^{(0)} \delta_{q, 0}+\delta z_{q}^{\sigma}
$$

$$
\lambda_{q}=\lambda^{(0)} \delta_{q, 0}+\delta \lambda_{q}
$$

where the zeroth-order MF solution, $z_{\downarrow}^{(0)}=0$ and $z_{\uparrow}^{(0)}=\sqrt{1-\delta}$. Equation (16) tells us that quantum fluctuations of the magnetic "order parameter" are supposed to be small. Besides that, Eq.(17) implies that the constraint-generated gauge field is slowly fluctuating function around its MF constant value. Assuming this, one can substitute Eqs. (1617) into the SF action to perform the fermion integral (10) at one-loop level. In this way, one arrives at a purely bosonic effective action. Analyzing then the stability criterion for the remaining bosonic integral over $\delta z_{\sigma}$ and $\delta \lambda$, one can evaluate the critical value, $\delta_{c} \approx 0.7$, at which the zeroth-order $\mathrm{MF}$ theory becomes unstable against quantum fluctuations.

However, this value of $\delta_{c}$ is too much large to agree with the recent results of the variational Monte Carlo studies $^{12}\left(\delta_{c}=0.38\right)$ and small cluster calculations ${ }^{13}$ $\left(\delta_{c}=0.22\right)$. Note that the smallest value of $\delta_{c}$ obtained so far is $\delta_{c}=0.17^{14}$. This MF value also contradicts the exact result obtained in infinite space dimension 27 .

The one-loop MF result can be improved, in principle, by imposing the crucial NDO constraint prior to the MF calculations. Surprisingly this is not the case. On the contrary, as we show in the next section an improved MF one-loop theory predicts exactly the same result that follows directly from the zeroth-order MF approximation: the FM phase is stable in the whole doping range, $0 \leq \delta<1$. This apparently indicates that the MF theory based on the MF treatment of the spin degrees of freedom affects the physics of the problem in a qualitative way. Within that approach, the emergent long-range ferromagnetic order appears to be just an artifact of the MF approximation, rather than a dynamically generated effect. 


\section{SLAVE-FERMION EFFECTIVE ACTION WITH THE EXPLICITLY RESOLVED NDO CONSTRAINT}

Let us turn back to the exact representation (10). As a first step to proceed, we rigorously resolve the constraint $\bar{\psi}_{i} \psi_{i}+\sum_{\sigma} \bar{z}_{i \sigma} z_{i \sigma}=1$. As a result, we arrive at the SF path-integral representation of the partition function that explicitly incorporates the NDO constraint.

The constraint can be explicitly resolved by making the identifications

$$
\begin{aligned}
z_{i \uparrow} & =\frac{e^{i \phi_{i}}}{\sqrt{1+\bar{z}_{i} z_{i}+\bar{\xi}_{i} \xi_{i}}}, z_{i \downarrow}=\frac{z_{i} e^{i \phi_{i}}}{\sqrt{1+\bar{z}_{i} z_{i}+\bar{\xi}_{i} \xi_{i}}}, \\
\psi_{i} & =\frac{\xi_{i} e^{i \phi_{i}}}{\sqrt{1+\bar{z}_{i} z_{i}+\bar{\xi}_{i} \xi}},
\end{aligned}
$$

with the variables $z_{i}, \xi_{i}$ and $\phi_{i}$ being free of any further constraints.

Note that the local gauge transformation (7) reasserts itself in the form, $\phi_{i} \rightarrow \phi_{i}+\theta_{i}$. In contrast, the projected variables

$$
z_{i}=z_{i \downarrow} / z_{i \uparrow}, \quad \xi_{i}=\psi_{i} / z_{i \uparrow}
$$

are seen to be manifestly gauge invariant. As we already mentioned, this gauge symmetry is a consequence of the redundancy in parametrizing the electron operator in terms of the auxiliary boson/fermion fields. The gauge ambiguity related with the redundancy of the SF representation is now expressed by a single variable $\phi_{i}$.

The domain of the flat measure in (10) that involves the spin up bosonic fields can be rewritten at every lattice site as $D \bar{z}_{i \uparrow} D z_{i \uparrow}=D\left|z_{i \uparrow}\right|^{2} D \phi_{i}$. The $\left|z_{i \uparrow}\right|^{2}$ field can easily be integrated out from Eq.(10) thanks to the constraint (12). Since the action in (10) is $U(1)$ gauge invariant and hence independent of $\phi_{i}$, the integration over $\phi_{i}$ results merely in the appearance of some numerical factor (volume of the gauge group) that can be taken care of by a proper normalization of the partition function. For the remaining integration we have (the site dependence for the moment being suppressed),

$$
D z_{\downarrow} D \bar{z}_{\downarrow} D \psi D \bar{\psi}=\operatorname{sdet}\left\|\frac{\partial\left(z_{\downarrow}, \bar{z}_{\downarrow}, \psi, \bar{\psi}\right)}{\partial(z, \bar{z}, \xi, \bar{\xi})}\right\| d z d \bar{z} d \xi d \bar{\xi}
$$

The Jacobian of this transformation (superdeterminant) was evaluated $\operatorname{in}^{28}$ to yield

$$
\operatorname{sdet}\left\|\frac{\partial\left(a_{\downarrow}, \overline{a_{\downarrow}}, f, \bar{f}\right)}{\partial(z, \bar{z}, \xi, \bar{\xi})}\right\|=\frac{1}{1+|z|^{2}+\bar{\xi} \xi} .
$$

Putting everything together we get a new representation for the SF partition function (10) with the local NDO constraint being explicitly resolved to give

$$
Z=\int D \mu(z, \xi) e^{S(z, \xi)}
$$

where

$$
D \mu(z, \xi)=\prod_{j, t} \frac{d \bar{z}_{j}(t) d z_{j}(t)}{2 \pi i} \frac{d \bar{\xi}_{j}(t) d \xi_{j}(t)}{1+\left|z_{j}\right|^{2}+\bar{\xi}_{j} \xi_{j}}
$$

stands for the measure with the boundary conditions, $z_{j}(0)=z_{j}(\beta), \xi_{j}(0)=-\xi_{j}(\beta)$. The action now reads

$$
\begin{aligned}
S(z, \xi)= & \frac{1}{2} \sum_{j} \int_{0}^{\beta} \frac{\dot{\bar{z}}_{j} z_{j}-\bar{z}_{j} \dot{z}_{j}+\dot{\bar{\xi}}_{j} \xi_{j}-\bar{\xi}_{j} \dot{\xi}_{j}}{1+\left|z_{j}\right|^{2}+\bar{\xi}_{j} \xi_{j}} d t \\
& -\int_{0}^{\beta} H^{c l} d t .
\end{aligned}
$$

The first part of the action (21) is a purely kinematic term that reflects the geometry of the underlying phase space while the classical Hamiltonian becomes,

$$
H^{c l}=t \sum_{i j} \frac{\bar{\xi}_{j} \xi_{i}\left(1+z_{j} \bar{z}_{i}\right)+H . c .}{\left(1+\left|z_{i}\right|^{2}+\bar{\xi}_{i} \xi_{i}\right)\left(1+\left|z_{j}\right|^{2}+\bar{\xi}_{j} \xi_{j}\right)}
$$

The new set of the gauge invariant variables, $(z, \xi)$, explicitly resolve the NDO constraint at the apparent expense of a more complicated compact phase space for the projected electron operators.

Finally, we make a change of variables to decompose the full measure given by (20) into the product of the conventional spin and fermion measures,

$$
\begin{aligned}
D \mu_{\text {spin }}(\bar{z}, z) & =\prod_{j, t} \frac{d \bar{z}_{j}(t) d z_{j}(t)}{2 \pi i\left(1+\left|z_{j}(t)\right|^{2}\right)^{2}}, \\
D \mu_{\text {fermion }}(\bar{\xi}, \xi) & =\prod_{j, t} d \bar{\xi}_{j}(t) d \xi_{j}(t),
\end{aligned}
$$

respectively. Such a reparametrization can be taken to be

$$
z_{i} \rightarrow z_{i}, \xi_{i} \rightarrow \xi_{i} \sqrt{1+\left|z_{i}\right|^{2}}
$$

Up to an inessential factor which redefines the chemical potential, we get

$$
D \mu \rightarrow D \mu_{\text {spin }}(\bar{z}, z) \times D \mu_{\text {fermion }}(\bar{\xi}, \xi),
$$

and the effective action becomes

$$
\begin{aligned}
S & =\sum_{i} \int_{0}^{\beta} i a_{i}(\tau) d \tau-\sum_{i} \int_{0}^{\beta} \bar{\xi}_{i}\left(\partial_{\tau}+\mu+i a_{i}\right) \xi_{i} d \tau \\
& -\int_{0}^{\beta} H^{c l} d \tau
\end{aligned}
$$

This action involves the $\mathrm{U}(1)$-valued connection one-form of the magnetic monopole bundle that can formally be interpreted as a spin "kinetic" term,

$$
i a=-\left\langle z\left|\partial_{t}\right| z\right\rangle=\frac{1}{2} \frac{\dot{\bar{z}} z-\bar{z} \dot{z}}{1+|z|^{2}},
$$


with $|z\rangle$ being the $\mathrm{su}(2)$ coherent state (see Appendix). This term is also frequently referred to as the Berry connection. The dynamical part of the action takes the form

$$
H^{c l}=t \sum_{i j}\left(\bar{\xi}_{j} \xi_{i}\left\langle z_{i} \mid z_{j}\right\rangle+H . c .\right) .
$$

Here $\left\langle z_{i} \mid z_{j}\right\rangle$ stands for an inner product of the su(2) coherent states,

$$
\left\langle z_{i} \mid z_{j}\right\rangle=\frac{1+\bar{z}_{i} z_{j}}{\sqrt{\left(1+\left|z_{j}\right|^{2}\right)\left(1+\left|z_{i}\right|^{2}\right)}} .
$$

The classical image of the on-site electron spin opertor (13) reduces to

$$
\vec{Q}_{i}^{c l}=\vec{S}_{i}^{c l}\left(1-\bar{\xi}_{i} \xi_{i}\right)
$$

where $\vec{S}^{c l}$ is given by Eqs. (51).

The action (25) is invariant under the global $\mathrm{SU}(2)$ rotations that now take the form

$$
z_{i} \rightarrow \frac{u z_{i}+v}{-\bar{v} z_{i}+\bar{u}}, \quad \xi_{i} \rightarrow e^{i \zeta_{i}} \xi_{i}, \quad a_{i} \rightarrow a_{i}-d \zeta_{i}
$$

where

$$
\zeta_{i}=-i \log \sqrt{\frac{-v \bar{z}_{i}+u}{-\bar{v} z_{i}+\bar{u}}}, \quad\left(\begin{array}{ll}
u & v \\
-\bar{v} & \bar{u}
\end{array}\right) \in \mathrm{SU}(2) .
$$

Equations $(25,26)$ provide a rigorous representation of the effective action of the $U=\infty$ Hubbard model. One can therefore wonder if these equations lead to the expected result if one nullifies the $t_{i j}$ hoppings. In this limit the infinite $U$ Hubbard model (8) reduces to the exactly solvable reduced Hamiltonian:

$$
H=\mu \sum_{i \sigma}\left(1-\tilde{c}_{i \sigma}^{\dagger} \tilde{c}_{i \sigma}\right)=\sum_{i} X_{i}^{00}
$$

where the Hubbard operator $X^{00}$ is represented by the diagonal $3 \times 3$ matrix with eigenvalues 0,0 and 1 . As a result, the partition function reduces simply to:

$$
Z=2+e^{-\beta \mu} .
$$

As a check of the validity of our representation our equations (25|26) should also recover this result.

To see that this is indeed the case, let us consider the on-site action (25) at $t=0$,

$$
S=\int_{0}^{\beta} i a(\tau) d \tau-\int_{0}^{\beta} \bar{\xi}\left(\partial_{\tau}+\mu+i a\right) \xi d \tau .
$$

By a gauge transformation the potential $a(\tau)$ can be brought into a time independent form,

$$
a \rightarrow a-\dot{\phi}=\frac{1}{\beta} \int_{0}^{\beta} a d \tau,
$$

where

$$
\phi(\tau)=-\frac{\tau}{\beta} \int_{0}^{\beta} a d s+\int_{0}^{\tau} a d s .
$$

Note that $\phi(0)=\phi(\beta)$. The effective action then becomes

$$
S=\int_{0}^{\beta} i a(\tau) d \tau-\int_{0}^{\beta} \bar{\xi}\left(\partial_{\tau}+\bar{\mu}\right) \xi d \tau,
$$

where $\bar{\mu}=\mu+\frac{1}{\beta} \int_{0}^{\beta} i a d \tau$. The partition function is now given by the path integral

$$
Z=\int D \mu e^{S}
$$

where the measure factor is represented by Eq.(24). Integrating out fermions yields

$$
Z=\int D \mu_{\text {spin }} e^{\int_{0}^{\beta} i a(\tau) d \tau}\left(1+e^{-\beta \bar{\mu}}\right) .
$$

Since

$$
\int D \mu_{\text {spin }} e^{\int_{0}^{\beta} i a(\tau) d \tau}=\operatorname{Tr}_{\text {spin }} \hat{I}=2,
$$

and $\int D \mu_{\text {spin }}=1$, one finally gets $Z=2+e^{-\beta \mu}$, which is indeed what it should be desired.

\section{A. Improved MF theory: zeroth-order approximation}

In this approximation the bosonic spin variable $z_{i}(t)$ takes on a macroscopic value $z^{(0)}$. Since $\left\langle z^{(0)} \mid z^{(0)}\right\rangle=1$, the spinless fermion MF Hamiltonian (26) reduces to the representation

$$
H^{c l}=t \sum_{i j}\left(\bar{\xi}_{j} \xi_{i}+H . c .\right) .
$$

This zeroth-order Hamiltonian does not depend on $z^{(0)}$ whose value merely determines a direction of the total electron magnetic moment,

$$
\vec{Q}_{M F}=\vec{S}_{c l}\left(\bar{z}^{(0)}, z^{(0)}\right)(1-\delta),
$$

with the explicit representation of the spin moment $S_{c l}(\bar{z}, z)$ being given in Appendix.

¿From now on we take the total electron magnetic moment aligned along the $z$-axis. To achieve this, one needs to set $z^{(0)}=0$ (see Appendix). The Hamiltonian (29) along with Eq.(30) describes a fully polarized ferromagnet for any hole concentration $\delta<1$. Therefore, the MF theory that takes care of the NDO constraint at the outset, produces naturally, at zeroth-order approximation, nearly the very same result that is produced by the zeroth-order MF theory (14) which only treats the NDO constraint globally. Note, however, that in the 
representation (29), in contrast to Eq.(15), there is no renormalization of the fermionic bandwidth. Again, one sees that due to the NDO constraint, the MF treatment of the spin dynamics, $z_{i}(t)=z^{(0)}$, automatically drives the system into an ordered phase. Regardless of any specific value of the constraint-free spin variable $z^{(0)}$, the system always stays in the ordered ferromagnetic phase as dictated by Eq. (30). The "order parameter" $z^{(0)}$, once again, fails to describe a phase transition out of the ferromagnetic phase.

\section{B. Improved MF theory: one-loop approximation}

In the present subsection we derive the one loop approximation for the effective MF spin action, with the local NDO constraint built in from the outset.

To this end we take into account the Gaussian fluctuatins of the bosonic spin variables around their MF value,

$$
z_{i}(t)=z^{(0)}+\delta z_{i}(t)=\delta z_{i}(t)
$$

and expand the action (25) up to the quadratic order in the new $\delta z_{i}$ variables. The fermionic path-integral can then be evaluated to this order explicitly and we end up with the following one-loop spin MF effective action,

$$
S=S_{0}+\Delta S
$$

where $S_{0}$ represents the zeroth-order MF action, whereas

$$
\Delta S=\operatorname{Tr} \delta(i a)+\operatorname{Tr} G_{(t)} \delta \Sigma .
$$

Here

$$
\delta\left(i a_{i}\right)=\frac{1}{2}\left(\dot{\bar{\delta}} \bar{z}_{i} \delta z_{i}-\bar{\delta} \bar{z}_{i} \delta \dot{z}_{i}\right)
$$

is a linearized spin kinetic term,

$$
\delta \Sigma_{i j}=-t_{i j}\left(\delta \bar{z}_{j} \delta z_{i}-\frac{1}{2}\left|\delta z_{i}\right|^{2}-\frac{1}{2}\left|\delta z_{j}\right|^{2}\right),
$$

and

$$
G_{(t)}^{-1}=\left(\partial_{t}-\mu-t_{i j}\right)
$$

is a zeroth-order MF Green function. The trace has to be carried out in both space and time indices.

In the momentum space the action (33) reads

$$
\begin{aligned}
\Delta S & =\frac{1}{2} \sum_{\vec{q}} \int_{0}^{\beta}\left(\dot{\bar{z}}_{\vec{q}} \delta z_{\vec{q}}-\delta \overline{z_{\vec{q}}} \dot{\delta} z_{\vec{q}}\right) d \tau \\
& -\sum_{\vec{q}} \int_{0}^{\beta} \omega_{\vec{q}} \delta \bar{z}_{\vec{q}} \delta z_{\vec{q}} d \tau,
\end{aligned}
$$

where

$$
\omega_{\vec{q}}=\frac{1}{N} \sum_{\vec{p}} f_{\vec{p}}\left(t_{\vec{p}+\vec{q}}-t_{\vec{p}}\right),
$$

and $f_{\vec{p}}=\left(e^{\beta\left(t_{\vec{p}}+\mu\right)}+1\right)^{-1}$ is the Fermi distribution function. The action (34) corresponds to the bosonic spinwave Hamiltonian,

$$
H=\sum_{\vec{q}} \omega_{\vec{q}} b_{\vec{q}}^{\dagger} b_{\vec{q}}, \quad\left[b_{\vec{q}^{\prime}}^{\dagger}, b_{\vec{q}}\right]=\delta_{\vec{q}^{\prime}, \vec{q}} .
$$

with the ferromagnetic spin-wave dispersion relation, $\omega_{\vec{q}} \propto \vec{q}^{2}, \vec{q} \rightarrow 0$.

These quantum fluctuations cannot destabilize the zeroth-oreder MF solution, since the excitation spectrum $\omega_{\vec{q}}$ is a non-negative function of the hole concentration $\delta$, provided $t_{\vec{p}}=t_{-\vec{p}}$. This can be proven in the following way: ${ }^{29}$

$$
\begin{aligned}
\omega_{\vec{q}} & =\frac{1}{N} \sum_{\vec{p} \in B Z} f_{\vec{p}}\left(t_{\vec{q}+\vec{p}}-t_{\vec{p}}\right) \\
& =\frac{1}{2} \frac{1}{N} \sum_{\vec{p} \in B Z} f_{\vec{p}}\left(t_{\vec{q}+\vec{p}}-t_{\vec{p}}\right)+\frac{1}{2} \frac{1}{N} \sum_{\vec{p} \in B Z} f_{\vec{p}}\left(t_{\vec{q}+\vec{p}}-t_{\vec{p}}\right)
\end{aligned}
$$

In the second summation we substitute $\vec{p} \rightarrow-\vec{p}-\vec{q}$. In this way we get

$$
\begin{aligned}
\omega_{\vec{q}} & =\frac{1}{2} \frac{1}{N} \sum_{\vec{p} \in B Z} f_{\vec{p}}\left(t_{\vec{q}+\vec{p}}-t_{\vec{p}}\right) \\
& +\frac{1}{2} \frac{1}{N} \sum_{\vec{p} \in B Z} f_{-\vec{p}-\vec{q}}\left(t_{\vec{q}-\vec{p}-\vec{q}}-t_{-\vec{p}-\vec{q}}\right) \\
& =\frac{1}{2} \frac{1}{N} \sum_{\vec{p} \in B Z} f_{\vec{p}}\left(t_{\vec{q}+\vec{p}}-t_{\vec{p}}\right) \\
& +\frac{1}{2} \frac{1}{N} \sum_{\vec{p} \in B Z} f_{\vec{p}+\vec{q}}\left(t_{\vec{p}}-t_{\vec{p}+\vec{q}}\right) \\
& =\frac{1}{2} \frac{1}{N} \sum_{\vec{p} \in B Z}\left(f_{\vec{p}}-f_{\vec{p}+\vec{q}}\right)\left(t_{\vec{q}+\vec{p}}-t_{\vec{p}}\right)
\end{aligned}
$$

Since $f_{\vec{p}}$ is monotonically decreasing function of $t_{\vec{p}}$, the quantity $\left(f_{\vec{p}}-f_{\vec{p}+\vec{q}}\right)\left(t_{\vec{q}+\vec{p}}-t_{\vec{p}}\right)$ is always non-negative.

In fact, the spin-wave stability condition, $\omega_{\vec{q}}>0, \vec{q} \neq$ 0 , is only a necessary condition for the saturation of ferromagnetism ${ }^{30}$. However, it is possible to show that if the semiclassical analysis is assumed to be true, this also becomes a sufficient condition. Therefore, if the MF (semiclassical) decomposition of the spin amplitude (31) is taken for grant, the ensuing SF MF theory is spin-wave stable over the whole doping range.

There is, however, a rigorous result that states that for a large enough hole concentration the Nagaoka state posesses an instability ${ }^{21}$. This result is heavily based on the consideration that the spin degrees of freedom in the $U=\infty$ limit are in fact dynamical variables rather than nearly frozen spins in the background. As was discussed above, the MF treatment of the spin degrees of freedom automatically drives the system into the ordered FM phase. Although in this case the SF MF theory works well, it is applied to a substantially altered Hamiltonian 
that does not seem to bear much in common with the original problem.

\section{ONE-LOOP APPROXIMATION BEYOND MF THEORY}

In the preceeding section we derived the improved oneloop MF theory to treat the Nagaoka's phase. In the improved theory we imposed the NDO constraint rigorously from the beginning and, only after that, the MF approximation was applied to describe the dynamics of the spin degres of freedom. The improved theory predicts a stable FM phase over the whole doping range. At the same time, the standard SF MF theory that treats the NDO constraint globally at the zeroth-order and locally at the one-loop order tells us that the FM phase is stable over a finite though quite large doping range. Since the improved MF theory should in any event provide a better description, one may conclude that the predictions of the standard MF theory are not reliable and are just an artifact of the uncontrolled MF treatment.

In this sense, it is interesting to note, that in an earlier paper ${ }^{9}$ a variational principle was formulated in such a way that the NDO constraint was automatically built into the trial state vector which considers that all the electron spins except one are aligned. The remaining electron is kept frozen. It can then rigorously be shown that at $U=\infty$ the Nagaoka ferromagnet is always stable for any $\delta$. This qualitatively agrees with the result following from the improved one-loop MF theory.

However, if the remaining electron is also allowed to hop around, the FM ground state immediately becomes unstable for large enough doping ${ }^{21}$. All this indicates, that even the improved MF approximation is too restrictive in the sense that it qualitatively affects the physics described by the model by unnecessarily freezing the spin degrees of freedom. This leads to the physically incorrect conclusion of predicting the FM phase for the whole doping range ${ }^{31}$. It is evident that not only the NDO constraint needs to be fulfilled. It is also very important to treat the electron correlations encoded by that constraint in a proper way considering both charge and spin degrees of freedom on equal footing as dynamical variables. It would therefore be very appealing to address the problem of the thermodynamic properties of the Nagaoka phase starting right from the low-energy effective quantum spin Hamiltonian rather than from the quasi-classical MF spin effective action. Technically this approach is, however, quite complicated. In view of that we report in this section only some preliminary results.

Specifically, we derive the contribution to the lowenergy effective spin action of the $U=\infty$ Hubbard model up to the lowest nonvanishing order in the spin self-energy $\Sigma_{i j}=-t\left\langle z_{j} \mid z_{i}\right\rangle$ beyond the MF approxima- tion. To this end, we rewrite action (25) in the form

$$
\begin{aligned}
S & =i \sum_{i} \int_{0}^{\beta} a_{i}(\tau) d \tau \\
& +\sum_{i j} \int_{0}^{\beta} \bar{\xi}_{i}(\tau) G_{i j}^{-1}(\tau, \sigma) \xi_{j}(\sigma) d \tau d \sigma,
\end{aligned}
$$

where

$G_{i j}^{-1}(\tau, \sigma)=G_{(0) i j}^{-1}(\tau, \sigma)-i a_{i}(\tau) \delta_{i j} \delta(\tau-\sigma)+\Sigma_{i j}(\tau) \delta(\tau-\sigma)$,

with $\Sigma_{i j}=-t_{i j}\left\langle z_{j} \mid z_{i}\right\rangle$ and

$$
G_{(0) i j}^{-1}(\tau, \sigma)=\delta_{i j}\left(-\partial_{\tau}-\mu\right) \delta(\tau-\sigma) .
$$

The fermionic degrees of freedom can formally be integrated out to yield

$$
\begin{aligned}
& \int D \bar{\xi} D \xi \exp \left(\sum_{i j} \int_{0}^{\beta} \bar{\xi}_{i}(\tau) G_{i j}^{-1}(\tau, \sigma) \xi_{j}(\sigma) d t d s\right) \\
= & \exp \operatorname{Tr} \log G^{-1} \\
= & \exp \left(\operatorname{Tr} \log G_{(0)}^{-1}+\operatorname{Tr} \log \left(1-G_{(0)} i a+G_{(0)} \Sigma\right)\right)(39)
\end{aligned}
$$

Here the trace has to be carried out over both space and time indices. Calculating explicitly the contribution coming from the zero order Green's function, it yields:

$$
\begin{aligned}
Z_{0}: & =Z_{t=a=0}=\exp \left(\operatorname{Tr} \log G_{(0)}^{-1}\right) \\
& =\left(2 \cosh \frac{\mu \beta}{2} e^{-\frac{\mu \beta}{2}}\right)^{N},
\end{aligned}
$$

which reproduces the exact result for the partition function of $\mathrm{N}$ noninteracting spinless fermions,

$$
Z_{0}=\operatorname{tr} e^{-\mu \int_{0}^{\beta} \sum_{i} f_{i}^{\dagger} f_{i}}
$$

We now evaluate the contributions of the self-energy $\Sigma_{i j}$ and of the gauge potential $a_{0}(i)$ up to the first nonvanishing order. This can be done in the usual way by making a loop expansion in the trace 32,33 . Here we are interested in the lowest order contribution that survives in the low-energy and long-wavelength limit. This limit consists in expanding the one-loop contribution up to first order in $\partial_{\tau}$ and up to the second order in $\vec{R}_{i}-\vec{R}_{j}$ implying that, eventually, we will set $i \rightarrow j$. This amounts to the so-called gradient expansion corresponding to the low-energy and long-wavelength limit of the action. We obtain in this way

$$
\begin{aligned}
& \operatorname{Tr} \log \left(1-G_{(0)} i a+G_{(0)} \Sigma\right)= \\
& -\operatorname{Tr}\left(G_{(0)} i a\right)-\operatorname{Tr}\left[\frac{1}{2}\left(G_{(0)} \Sigma G_{(0)} \Sigma\right)+\mathcal{O}\left(\Sigma^{3}\right)\right]
\end{aligned}
$$

Note that $\operatorname{Tr}\left(G_{(0)} i a G_{(0)} \Sigma\right)=0$ since $\Sigma_{i i}=0$. Note that Eq.(40) is invariant under global rotations. This immediately follows from the transformation law of the given 
by Eq.27) accompanied by the similar transformations from the $\Sigma_{i j}$ 's:

$$
\Sigma_{i j} \rightarrow e^{-i \zeta_{j}+i \zeta_{i}} \Sigma_{i j}
$$

The a-dependent term in Eq.(40) contributes to the action in the following way

$$
-\operatorname{Tr}\left(G_{(0)} i a\right)=-i \sum_{i} G_{(0) i}\left(0^{-}\right) \int_{0}^{\beta} a_{i}(\tau) d \tau,
$$

where $G_{(0) i}\left(0^{-}\right):=\lim _{\epsilon \rightarrow 0} G_{(0) i}(-\epsilon), \epsilon>0$ and

$$
G_{(0) i}(\tau)=\frac{e^{-\mu \tau}}{1+e^{\mu \beta}}-\theta(\tau) e^{-\mu \tau} .
$$

The explicit representation (41) tells us that

$$
\operatorname{Tr}\left(G_{(0)} i a\right)=\mathcal{O}\left(e^{-\mu \beta}\right), \quad \mu \beta>>1 .
$$

Let us now turn to the second term in Eq.(40). We get

$$
-\frac{1}{2} \operatorname{Tr}\left(G_{(0)} \Sigma G_{(0)} \Sigma\right)
$$

$=-\frac{1}{2} \sum_{i j} \int G_{(0) i}\left(t_{1}-t_{2}\right) \Sigma_{i j}\left(t_{2}\right) G_{(0) j}\left(t_{2}-t_{1}\right) \Sigma_{j i}\left(t_{1}\right) d t_{1} d t_{2}$.

Introducing new variables, $\tau=\frac{t_{1}-t_{2}}{2}, \eta=\frac{t_{1}+t_{2}}{2}$, and expanding the product $\Sigma_{i j}(\eta+\tau) \Sigma_{j i}(\eta-\tau)^{2}=$ $\Sigma_{i j}(\eta) \Sigma_{j i}(\eta)+\mathcal{O}(\tau)$ (this corresponds to the gradient expansion in imaginary time $\left.{ }^{33}\right)$, this, to lowest order, reduces to

$$
-\frac{1}{2} \sum_{i j} \int_{-\beta}^{\beta} G_{(0)}(\tau) G_{(0)}(-\tau) d \tau \int_{0}^{\beta} \Sigma_{i j}(\eta) \Sigma_{j i}(\eta) d \eta
$$

With the help of Eq.411) we get

$$
-\frac{1}{2} \int_{-\beta}^{\beta} G_{(0)}(\tau) G_{(0)}(-\tau) d \tau=\frac{\beta}{4} \frac{1}{\cosh ^{2}(\beta \mu / 2)} .
$$

Note that the energy scale is set by the chemical potential $\mu$, so that the low-energy limit takes the form $\mu \beta \gg 1$.

The effective spin action is then given by the sum of all the terms evaluated above:

$$
Z_{e f f} / Z_{0}=\int D \mu(z, \bar{z}) e^{S_{e f f}},
$$

where the $\mathrm{SU}(2)$ invariant measure is

$$
D \mu(z, \bar{z})=\prod_{i, t} \frac{d \bar{z}_{i}(t) d z_{i}(t)}{2 \pi i\left(1+\left|z_{i}\right|^{2}\right)^{2}}
$$

and the effective action:

$$
\begin{aligned}
S_{e f f} & =i \sum_{i} \int_{0}^{\beta} a_{i}(\tau) \\
& +\sum_{i j} \int_{0}^{\beta} \frac{J_{i j}^{e f f}}{2}\left|\left\langle z_{i} \mid z_{j}\right\rangle\right|^{2},
\end{aligned}
$$

with the long-wavelength limit $(i \rightarrow j)$ being implicit throughout our calculation. This action describes the $\mathrm{SU}(2)$ invariant ferromagnetic Heisenberg model with the effective coupling (see Appendix)

$$
-J_{i j}^{e f f}=-\left(\beta\left|t_{i j}\right|^{2}\right) /\left(2 \cosh ^{2} \frac{\beta \mu}{2}\right) \leq 0 \text {. }
$$

Since

$$
J_{i j}^{e f f}=\mathcal{O}\left(e^{-\mu \beta}\right), \quad \mu \beta>>1
$$

this result tells us that the lowest order contribution to the free energy in $\Sigma$ shows no magnetic ordering, provided $\mu \beta \gg 1$. However, one can safely truncate the expansion of the effective action (40) at second order, provided $|t| \beta \ll 1$. This, however, does not generate a high-temperature expansion of the free energy. The point being that the temperature is supposed to be "high" compared to the overall energy scale factor $t$. However, it still might be low compared with the "intrinsic" energy scale which is set by $\mu$. Physically, the limit $\mu \beta \gg 1$ corresponds to a very small hole concentration ${ }^{34}$. Therefore, our result (47) provides us with a limited piece of information concerning the thermodynamic instability of the Nagaoka phase at finite temperature. It merely indicates that the FM order exponentially decays away from half filling at any finite temperature $T=1 / \beta \gg|t|$.

To address the issue of the thermodynamic instability of the Nagaoka phase at any $T \geq 0$ one should go back to the full series in the low-energy long-wavelength expansion of the fermionic determinant (40) and analyse further the dynamics of the spin variables as well. On the bipartite lattice with the nn interaction, that series can be summed up to yield the following contribution to the low-energy effective spin action:

$$
\Delta S=\frac{e^{-\mu \beta}}{2 \beta} \int_{0}^{\beta} d t \operatorname{Tr} \cosh (2 \beta \Sigma(t)), \quad \mu \beta>>1 .
$$

The next step is now to calculate the long-wavelength asymptotic of this representation, which is not a trivial follow up and it still is in progress. Note only that this result is strongly dependent on the space dimensionality. However, there exists a universal feature of the $U=\infty$ Hubbard model phase diagram that naturally emerges from Eq.(48): the paramagnetic state is thermodynamically stable at any finite temperature at $\delta=0$. This can be derived by considering the limit $\mu \rightarrow \infty$ in Eq. (48) at finite values of $\beta$ and $t$. This result agrees with the qualitative arguments presented in ${ }^{20}$. In contrast with that, the MF treatment continue to predict in this case a fully polarized FM state.

\section{CONCLUSION}

In this paper, we formulate the improved SF MF theory to describe the FM phase of the Nagaoka limit of 
the Hubbard model. It is clear that the physics behind the Nagaoka phase is controlled by strong electron correlations. Those correlations are in turn encoded into the NDO constraint. We improve the standard MF approach by taking the NDO constraint rigorously into consideration prior to the MF approximation. Once this is done we integrate out the fermionic degrees of freedom under the condition that the spin degrees of freedom are considered at the MF level. In this way we arrive at the one-loop MF theory of the Nagaoka phase that predicts a FM ordering over the whole doping range. This result agrees with earlier variational estimates which take the NDO constraint fully into account but leaves some of the spin degrees of freedom completely frozen. At the same time, the conventional SF MF theory, that treats the constraint at the MF level, predicts the FM phase over a finite though quite large doping range. This result of the standard MF theory thus appears just as an artifact of the uncontrolled treatment of the NDO constraint.

We show that the SF MF theory automatically drives the system into the FM ordered phase, and hence it cannot describe the underlying physics even in a qualitatively correct way. This happens because of the fact that the spin degrees of freedom are only considered quasiclassically. However, it is the quantum spin dynamics that ultimately determines the magnetic properties of the Nagaoka phase. Therefore, to get any physically reasonable result one should enforce both the NDO constraint rigorously and treat the dynamics of the spin degrees of freedom beyond the MF approximation.

As a first step in this direction, we derived the truncated low-energy long-wavelength effective spin action with the spin variables being treated beyond the MF approximation. This approximation corresponds to the expansion of the effective free energy in powers of a small parameter $|t| \beta$ close to half filling. It turns out that treating the spin dynamics beyond the MF approximation completely destroys the FM order predicted by the SF MF theory. Despite of that, this result does not prove the thermodynamic instability of the Nagaoka phase. To address this issue one should go back to the full nontruncated low-energy long-wavelength effective spin action as discussed in the previous sections of the paper. Nevertheless, we can add with certainty that the SF MF approach produces spurious results and is therefore not reliable for the description of the Nagaoka ferromagnetism.

\section{ACKNOWLEDGMENTS}

This work was partially supported by the Brazilian Ministry of Science and Technology and by CNPq.

\section{APPENDIX: SU(2) ALGEBRA AND} COHERENT STATES

Consider the $\mathrm{su}(2)$ algebra in the lowest $s=1 / 2$ representation:

$$
\left[S_{z}, S_{ \pm}\right]= \pm S_{ \pm}, \quad\left[S_{+}, S_{-}\right]=2 S_{z}, \quad \vec{S}^{2}=3 / 4 .
$$

Acting with the "lowering" spin operator $S^{-}$on the "highest weight" state $|\uparrow\rangle$ we get the normalized $\mathrm{su}(2)$ CS parametrized by a complex number $z$

$$
|z\rangle=\frac{1}{\sqrt{1+|z|^{2}}} \exp \left(z S^{-}\right)|\uparrow\rangle=\frac{1}{\sqrt{1+|z|^{2}}}(|\uparrow\rangle+z|\downarrow\rangle) .
$$

In the basis spanned by the vectors $|\uparrow\rangle,|\downarrow\rangle$ we have $S_{+}=|\uparrow\rangle\left|\left\langle\downarrow\left|, S_{-}=\right| \downarrow\right\rangle\right|\left\langle\uparrow\left|, S_{z}=\frac{1}{2}(|\uparrow\rangle|\langle\uparrow|-| \downarrow\rangle|\langle\downarrow|)\right.\right.$. The CS symbols of the $\mathrm{su}(2)$ generators are then easily evaluated to be $\left(S^{c l}:=\langle z|S| z\rangle\right)$ :

$$
\begin{aligned}
S_{+}^{c l}: & =\frac{z}{1+|z|^{2}}, \quad S_{-}^{c l}=\frac{\bar{z}}{1+|z|^{2}}, \\
S_{z}^{c l} & =\frac{1}{2} \frac{1-|z|^{2}}{1+|z|^{2}}, \quad \vec{S}_{c l}^{2}=1 / 4,\left(\vec{S}^{2}\right)_{c l}=3 / 4 .
\end{aligned}
$$

There is a one-to-one correspondence between the $\mathrm{su}(2)$ generators (49) and their CS (classical) symbols given by Eqs. (51). Given a quantum Hamiltonian $H=H(\vec{S})$, the corresponding imaginary time phasespace action takes on the form,

$$
\mathcal{A}_{s u(2)}(\bar{z}, z)=-\int_{0}^{\beta}\left\langle z\left|\frac{d}{d t}+H\right| z\right\rangle d t
$$

with the kinetic term being given by

$$
i a=-\left\langle z\left|\frac{d}{d t}\right| z\right\rangle=\frac{1}{2} \frac{\dot{\bar{z}} z-\bar{z} \dot{z}}{1+|z|^{2}}
$$

In particular, for the quantum $s=1 / 2$ Heisenberg model,

$$
H=J \sum_{i j}\left(\vec{S}_{i} \vec{S}_{j}-1 / 4\right)
$$

one gets

$$
H^{c l}=\frac{J}{2} \sum_{i j}\left(\left|\left\langle z_{i} \mid z_{j}\right\rangle\right|^{2}-1\right) .
$$

¿From the geometrical viewpoint, the su(2) coherent states $|z\rangle$ can be thought of as sections of the magnetic monopole bundle $P\left(S^{2}, U(1)\right)$, with the $\mathrm{U}(1)$ connection one-form, $i a$, frequently refereed to as the Berry connection. Base space of that bundle, two-sphere $S^{2}$, appears as a classical phase-space of spin, whereas its covariantly constant sections, $|z\rangle: \mathcal{D}_{t}|z\rangle:=\left(\partial_{t}+i a\right)|z\rangle=0$, form a Hilbert space of a quantum spin. 
1 S.E. Barnes, J. Phys. F 6, 1375 (1976); N. Read and D.M. Newns, J. Phys. C 16, 3273 (1983); P. Coleman, Phys. Rev. B 29, 3035 (1984); G. Baskaran and P.W. Anderson, Phys. Rev. B 37, 580 (1988).

2 D. Yoshioka, J. Phys. Soc. Jpn. 58, 1516 (1989); D.P Arovas and A. Auerbach, Phys. Rev. B 38, 316 (1988).

3 Chetan Nayak, Phys. Rev. Lett. 85, 178 (2000).

${ }^{4}$ H. Fukuyama, Prog. Theor. Phys. Suppl. 108, 287 (1992); C. L. Kane et al, Phys. Rev. B 41, 2653 (1990); S. Feng, Z.B. Su and L. Yu, Phys. Rev. B 49, 2368 (1994).

${ }^{5}$ E. Kochetov and A. Ferraz, Phys. Rev. B 70, 052508 (2004).

6 However, it is physically realizable in an optical lattice ${ }^{7}$.

7 D. Jaksch and P. Zoller, Ann. Phys. 315, 52 (2005).

8 Y. Nagaoka, Phys. Rev. 147, 392 (1966).

${ }^{9}$ P.Richmond and G. Rickayzen, J. Phys. C 2, 528, (1969).

10 J.A. Riera and A.P. Young, Phys. Rev. B 40, 5285 (1989).

11 M.W. Long, in "The Hubbard Model, recent results", ed. by M. Rasetti, World Scientific, (1991).

12 H. Yokoyama and H. Shiba, J. Phys. Soc. Japan 56, 3670 (1987); D. Dzierzawa and R. Frésard, Z. Phys. B91, 245 (1993).

13 E. Müller-Hartmann, Th. Hanisch and R. Hirsh, Physica B186-188, 834 (1993).

14 E.G. Goryachev and D.V. Kuznetsov, JETP Lett. 56, 203 (1992).

15 A. Suto, Commun. Math. Phys. 140, 43 (1991).

16 G.S. Tian, Phys. Rev. B 444444 (1991); J. Phys A 24, 2375 (1991).

17 W.O. Putikka, M.U. Luchini, M. Ogata, Phys. Rev. Lett. 69, 2288 (1992).
18 Hal Tasaki, Progress of Theoretical Physics 99, 489 (1998); cond mat/9712219v3.

19 F. Becca, S. Sorella, Phys. Rev. Lett. 86, 3396, (2001).

${ }^{20}$ Hyowon Park, K. Haule, C.A. Marianetti, and G. Kotliar, Phys. Rev. B 77, 035107 (2008).

21 B.S. Shastry, H.R. Krishnamurthy, and P.W. Anderson, Phys. Rev. B 41, 2375 (1990).

22 W. von der Linden, D Edwards, J. Phys. Cond Mat. 3, 4917 (1991),

23 A.G. Basile et al, Phys. Rev. B 41, 4842 (1990).

24 Th. Hanisch, G. S. Uhrig, and E. Muller-Hartmann, Phys. Rev. B 56, (1997).

25 D.J. Scalapino, R. L. Sugar, S. R. White, N. E. Bickers, R. T. Scalettar, Phys. Scr. T 27, T27, 101 (1989).

26 Daniel Boies, F.A. Jackson and A.-M.S. Tremblay, Int. J. Mod. Phys. B9, 1001 (1995)

27 P. Fazekas, B. Menge, and E. Müller-Hartmann, Z. Phys. B 78, 69 (1990).

28 A. Ferraz, E. Kochetov and M.Mierzejewski, Phys. Rev. B 73, 064516 (2006).

29 Dr. M.Mierzejewski, private communication.

30 Daniel Mattis, Phys. Rev. 130, 76 (1963).

31 This may lead to a scenario in which the FM could survive (at $U=\infty$ ) at any hole concentration, provided a Hamiltonian has a separation of charge and $\operatorname{spin}^{21}$.

32 A.M.J. Schakel, Mod. Phys. Lett. B 14, 927 (1990).

33 M. Cuoco and J. Ranninger, Phys. Rev. B 70, 104509 (2004).

34 X.Y. Zhang, Elihu Abrahams, and G. Kotliar, Phys. Rev. Lett. 66, 1236 (1991). 